\title{
The Impact of the Design of Hospitals on Hospital Hoteling, Healing Process and Medical Tourism
}

\author{
Arash Abinama ${ }^{1} \&$ Masoud Jafari ${ }^{2}$ \\ ${ }^{1}$ Department of Architecture, Savadkooh Branch, Islamic Azad University, Savadkooh, Iran \\ ${ }^{2}$ Department of Architecture, Roudehen Branch, Islamic Azad University, Roudehen, Iran \\ Correspondence: Arash Abinama, Flat 9., No. 6, Chakavak Blind Alley, West Ghanary Alley, Jolfa Str., \\ Seyedkhandan, Tehran, 1541745631, Iran. Tel: 98-912-621-2075. E-mail: arash.abinama@gmail.com
}

Received: May 9, 2015

Accepted: July 15, 2015

Online Published: October 31, 2015

doi:10.5539/mas.v9n12p43

URL: http://dx.doi.org/10.5539/mas.v9n12p43

\begin{abstract}
The design of hospitals is extremely important; any person may spend a part of his life in a hospital. Considering human interaction with the environment, psychological aspects of design and their impact on the moods of inpatients and their companions and medical team ergonomics and performance, the process of designing hospitals becomes more complex. The design of operational spaces in hospitals lack appropriate and functional properties in this regard. This paper considers the studies on the role of architecture and appropriate design on increasing the quality of the hospital hoteling, patients' satisfaction, life expectancy and improved healing process, considering the design of spaces for inpatients' companion and medical team. Furthermore, considering the potential and capacity of admitting foreign patients in Iran, the impact of these factors on medical tourism is also investigated. The objective of the present research is applied and is descriptive/analytical that considered various aspects of the problem from different perspectives. The findings of the research show that implementing the above standards in designing hospitals leads to increased satisfaction, improvement in inpatient morale, speedy healing process, and more relaxed patients' family and medical staff. In addition, the impacts of the above items will result in increased medical tourism.
\end{abstract}

Keywords: architectural design, hospital hoteling, medical tourism, increased life expectancy, design healing effects, modern hospital design

\section{Introduction}

Today, hospitals are one of the main centers providing health care and treatment for the population. Continuous changes in social and economic conditions as well as ever increasing medical and psychological knowledge and technologies have created the need for comprehensive changes in the delivery of health services in line with these developments. Reviewing the history of hospital construction, it is evident that design has been usually a reflection of developments in medical science and technology and limited to creating appropriate environment for the provision of health services. But over time, hospital design has had an immense impact on the development of medicine in twentieth century, showing the importance of design and architecture in specialized buildings in the history of development of hospitals. The old definitions of hospital maintain that it is a closed, sterile and isolated place with white interior and stone or brick exterior that young visitors are not allowed to enter. In addition, the chief task of the typical hospitals was providing medical services to patients. Under this old structure, the patients are dependent on the medical staff for spending the hard times of their treatment. Hence, the old structure can not meet the different needs of all patients (Elsayed Metwally Amr, 2013). The current methods of care and treatment are inadequate in providing the necessary services for the population, especially the elderly (Ham et al., 2012). The performance of health care organizations depends on several factors and to succeed in today's competitive environment of hospitals they should consider several factors including, reciprocal relationships among patients, their companions, physicians and medical team, as well as, the impact of hospital spaces on them. Hospital design can affect patient satisfaction, therefore, the competition for a design and structural architecture which results in more satisfaction of inpatients, their companions and medical team has become one of the main concerns and issues for competition among hospitals. Higher satisfaction among doctors leads to increased interest and attention to their patients and their treatment. There is a direct correlation between medical team satisfaction about their work environment and their handling of the 
patients. All of these instances, besides leading to increased patient comfort and satisfaction in health care environments, result in speedy healing process, reduced mortality rate and attraction of more visitors to the same medical center in future. On the other hand, shorter treatment time saves resources and more beds will be emptied for new inpatients. At the same time, patients' expenses are also reduced and they become more willing to come back to follow up their treatment. The results depend on observing appropriate principles in the design of hospitals in order to enhance inpatient comfort and sense of security, thereby enhancing the recovery process (Elsayed Metwally Amr, 2013). The kind of services offered by hospitals is a key factor in distinguishing between them. Indeed, we can compare hospitals to airlines in this respect, where good food, active and attentive crew, and pleasant environment all affect passenger satisfaction of the total services provided (Newhouse, 1994). On the other hand, the importance of medical tourism doubles for Iran considering the geopolitical status of Iran and its positioning among countries with inadequate medical equipment and facilities and/or lower level of medical knowledge and specialized physicians. Today in Iran and the world, it is one of the most rapidly growing sectors of the tourism industry and it is applicable in employment and economic areas, as well as a means to introduce Iranian culture to overseas. Iran, due to its climate and positioning, has the potential to become a hub for medical tourism in the region and even Asia. It is worth noting that now, due to the lower cost of treatment, many Iranians living abroad travel to Iran to do some of their treatments, especially dental procedures and general surgery.

Based on the current evidence, quality patient care and treatment process is linked to increased returns. According to Tay (Tay, 2003), in addition to medical facilities and clinical workforce, geographical location and building design is also a criterion for attracting patients to hospitals. Considering the listed evidences, the design and architectural style of hospitals, along with other health factors, are among the most impressive features in speeding healing process that can lead to lower hospitalization period, increased rate of next visits, development of medical tourism, and Iran becoming an outstanding center in the treatment of foreign patients in the region.

\section{Hospital Hoteling}

According to the state Health System Transformation Project, hoteling involves issues related to inpatient well-being including inpatient bed, inpatient room, appropriate spaces for inpatient companions, facilities such as TV, sleepwear, and everything that leads to well-being of inpatients and their companions; peace and comfort must be considered and addressed as hoteling services, too. Patients base their judgment on what they see, issues such as bathroom, bedding, sleepwear, wall paint, room space, welfare environment, and the accommodations for companions; even it is reflected in their food. And this cannot be assessed unless by asking the patients and their companions (Taherizadeh, 2014).

\section{Medical Tourism}

One of the major challenges for hospitals is to attract more patients and compete with other medical centers. At the moment, attracting tourists for treatment is of particular importance. Medical tourism has created a variety of opportunities and challenges for hospitals. They can attract patients adopting an appropriate approach to take advantage of their maximum potential (Nasiripour, 2011). Medical tourism is a process in which people travel to other countries to enjoy healthcare services (EI Taguri, 2007).

According to a joint research by York University, Sheffield Hospital, and Birmingham University, medical tourism is the provision and use of healthcare services where individuals travel outside of their own countries for promoting their physical and mental status using natural or medical facilities (Lunt et al., 2012).

Medical tourism, which is sometimes called health and healing tourism, refers to the process by which people from all over the world travel to other countries to benefit from healthcare services, and at the same time, they visit new places, sights and attractions in other countries. Medical tourism is very attractive economically. Tourists who travel to other countries for the treatment are often from industrialized countries such as United States, Canada, Great Britain, Western Europe, Australia and the Mediterranean countries. But as time went by, more and more people joined them from different countries who seek treatment at affordable prices in countries that have their favorite tourist attractions (Nedelea \& George, 2008). Various causes are behind medical tourism, which will be discussed in what follows in general and in Iran in particular.

\subsection{Factors Affecting Medical Tourism}

In addition to the high cost of treatment in some countries, issues such as the long waiting times for treatments like surgery, which sometimes reaches six months to two years, have made people to resort to medical tourism for their own or their relatives' treatment. On the other hand, insurance providers do not cover all cases required by people, and that is a strong incentive for people in rich countries for opting for medical tourism. It is likely 
that in the near future, insurance companies will encourage patients to use medical tourism to save on costs. Thus, in order to attract more tourists, hospitals in developing countries also offer therapeutic-recreational touristic packages, including therapy, accommodation, tourism services and visiting natural sights in the destination country. The range of such services in a country like India has developed in many fields such as naturopathic, homeopathic and yoga treatments (http://www.tram-research.com/MedicalTourism.PDF ).

\subsection{Iran's Status Regarding Medical Tourism}

Iran is among top countries having the potential for medical tourism, yet it ranks low in terms of performance. Tourism industry has made remarkable progress in countries such as Thailand, Singapore and Malaysia, despite the fact that Iran is in a desirable status in medicine, traditional medicine and medical facilities. According to a report by Qazvin University of Medical Sciences and Health Services, competencies of employees in the medical profession, modern technologies, international standards, healthcare economics, healthcare costs, and internal regulations of countries are among important factors promoting medical tourism. In all four cases mentioned above, Iran has more capabilities compared to the neighboring countries and in the Middle East. (http://www.qums.ac.ir/portal/home/?102302/)

\section{The Impact of Design and Architecture on Improved Healing Process}

When designing health facilities or renovating and re-designing existing buildings, three important points must be considered: design priorities, elements influencing the design, and its sustainability. Effective use of these elements in designing health facilities will promote the impact of such places in patient recuperating. In this way, we will have a holistic and coordinated view of life, which in turn, results in success and positive feedback from patients, their relatives and clinical workforce (Elsayed Metwally Amr, 2013). According to recent studies conducted in the Cochrane Library, environmental factors in hospitals such as sounds, images, smells, air quality, lighting, as well as design affect treatment and recuperation (Drahota, 2012).

Environment has a crucial role in the process of healing. Seunghae (2011) say that:

In this competitive healthcare market with growing patient consumerism, it is important for healthcare providers to understand what patients and families experience in their facilities how they perceive healthcare service quality, and what impacts those perceptions in order to satisfy and exceed patients' wants and needs. (p.61)

Therefore, designers should consider all of these aspects to provide design that is more suitable for the patients and their relatives.

People may need different facilities at their disposal. This depends on many factors such as their age, their abilities and the physical problems they may have. Hence, designers should provide more flexible designs to make the environment more comfortable and the facilities more accessible for different people with different needs. (Maguire, 2014)

\subsection{Design Priorities}

Design priorities, in general, involve finding appropriate answers to questions posed by architects and designers; finding design elements that cause therapeutic effects by the environment. Appropriate designing has a direct positive impact on administrators, caregivers, family members, and especially patients (Elsayed Metwally Amr, 2013). Answers to these questions are then used as a reference for the final design of hospitals. The design priorities should not be limited to certain cases, yet they must include the following three important issues:

- What are the current and future needs?

- How can we provide flexible interior and exterior designs which are friendly enough and acceptable to patients, relatives, visitors and the doctors and the medical team?

- How can we provide an elegant architecture appropriate for urban context without using strange forms and unnecessary complexity? And how to coordinate with the surrounding urban context and culture?

\subsection{Sustainability}

The second major factor in designing health facilities is their sustainability, that is, how architects should take moral responsibility for the environment. This sense of responsibility must be reflected in designing elements of the buildings; elements including preserving natural resources such as water, providing quality air for indoor spaces, reliable energy supply, as well as using sustainable building material. Sustainability is no longer an option but a necessity to observe and pursue in order to protect the environment. 


\subsection{Design Elements}

The theory of design elements is summarized in Figure 1. The level and manner of usage of these elements depends on several factors including, architect and designer views, urban context of the area, culture of the region, and it is not necessarily in the same order as shown in Figure 1. These elements are mentioned due to successful experiences gained in the design of hospitals. Thus, the elements affecting the design fall into three main groups: light, privacy, and circulation.

Their secondary variables are: nature, timeless, tradition, security, way-finding, contemporary, elegance, and artificial. It should be noted that the order of use of these elements can be changed, and it depends on the area, they also can be changed according to the external factors.

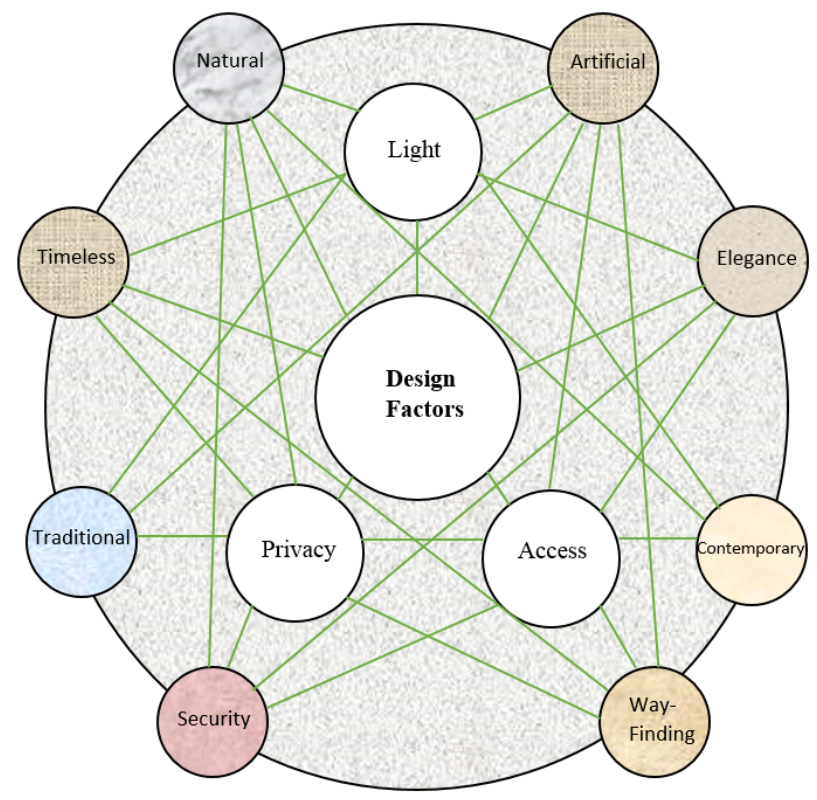

Figure 1. Theory of Elements Affecting Design (Pellitteri, Belvedere, 2010; Dietrich, 2004)

\subsubsection{Light}

Light is a primary element in the design of healthcare facilities and it is recommended to use natural light as much as possible. Thus, all measures must be considered to design spaces in a way that using natural light is maximized in all possible areas based on their function.

\subsubsection{Access}

Another primary element in designing healthcare facilities is Access. Floor surface design must be simple so that it would be possible to combine and implement various plans according to the needs. Furthermore, a clear separation between different medical wards, a controlled communication between them, as well as, separating visitors, patients and doctors are among other key features that must be considered. Each wall must be designed in a way that it would be possible to move it from one place to another without losing the apparent quality during re-designing.

\subsubsection{Privacy}

Another primary element in the design is observing privacy in the use of health facilities. In fact, the privacy of patients, their companions and clinical workforce must be considered in design. Privacy is of particular importance in the culture of certain societies, especially religious countries. An important point is that using the word "primary" for the above mentioned elements does not reduce the value of secondary variables. These secondary elements may be converted into primary design elements depending on the function and type of building. For example, security and tradition or any other secondary design elements are important features that are embedded in functional healthcare facilities. The aim of categorizing design ideas and elements is to make sure that everything is taken into account and properly applied in the design of healthcare facilities for offering the intended services. Providing high quality health and healing facilities should not be restricted to patients, but successful medical facility designs must also target relatives of patients and medical personnel. It is natural for 
inpatients to have many visitors, who may stay with the inpatient for a longer time. And the medical team usually works for long hours, cutting their social life to attend the patients; they also need a place to relax and refresh their body and soul in order to be able to efficiently tackle the task of patient care and follow-up (Pellitteri \& Belvedere, 2010; Dietrich, 2004). Today's societies are full of various diseases. Therefore, there is a need for appropriate designs and building more health facilities to be used to treat patients. Observance of abovementioned primary and secondary elements, regarding the successful experiences implemented in Middle Eastern Medical Center, had a major impact on the healing process, and patient, companion and medical team satisfaction; contributing to medical tourism, employment and higher revenues.

\section{Satisfaction}

Hospital directors in Asia have recognized the fact that patient satisfaction is a strategic variable affecting patient attraction, and thus, the survival of care centers in long run. In a research in Asia, data was obtained from 33 patients; patient satisfaction was analyzed based on the services provided as well as demographic and psychological variables (Dayasiri \& Lekamge, 2010; Farmahini Farahani et al., 2014). The data was obtained through interviews and questionnaires completed by patients and their companions. Demographic factors such as age, gender, literacy, and socio-economic status was influential on patients' satisfaction about care quality. The extent of influence was not equal in all Asian countries under study. Factors related to health service providers such as type of hospital, physical equipment, as well as communication between patients and health professionals also affect the level of patient satisfaction. The extent of this impact was different in various Asian countries (Dayasiri \& Lekamge, 2010; Farmahini Farahani et al., 2014).

For continuous quality promotion in health centers the use of timely feedback from patients and their relatives is essential. As patients are the main customers of care centers and hospitals, their satisfaction in evaluating offered services is of particular importance. The primary criterion for care and care environment and high quality services must be overall satisfaction of patients. Recognizing and considering the factors contributing in patient satisfaction is essential for every Asian country in order to promote quality. Therefore, it is necessary to recognize the key factors. Satisfaction factors, variables such as the characteristics of the patients, patients' expectations, their satisfaction, characteristics of medical centers, received care, and care experience were involved in positive assessment of the health centers and as a result reporting satisfaction and rating of the centers (Perneger, 2004). Since the first studies in the 1960s regarding patient satisfaction (Newsome \& wright, 1999), many studies have been done on this topic. It is estimated that more than 15000 commercial and academic papers were published in over 2 decades in this field before 2000 (Peterson \& Wilson 1992). It should be noted that the studies conducted in Asian countries are limited. Health care has a special status in ensuring continuous improvement of socio-economic status. Impairment of health sector leads in an evil circle that causes disease and poverty in society. Therefore, receiving constant feedback from patients to improve current situation is of particular importance. Figure 2 illustrates the theoretical model of patient satisfaction in the Oxford Journal:

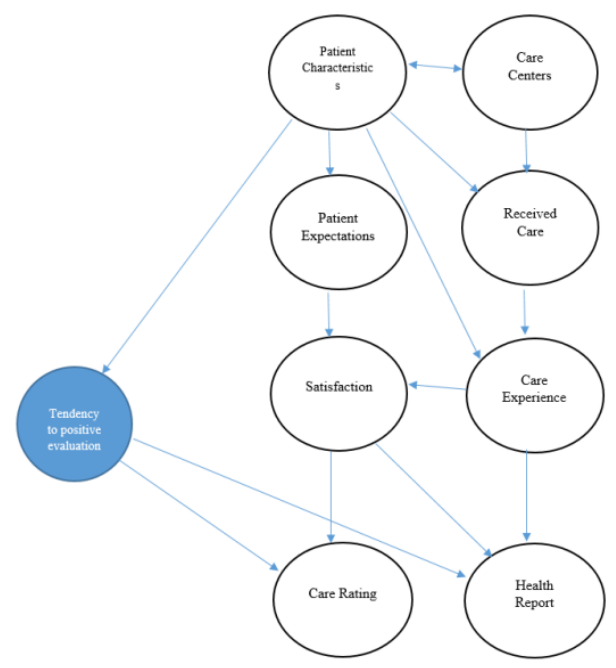

Figure 2. The theoretical model of patient satisfaction in the Oxford Journal (Perneger, 2004) 


\subsection{Life Expectancy}

The ability to satisfy the patients is essential for many reasons. By eliciting these feedbacks hospitals can provide better treatment- and care-based services for patients. Improving service quality will increase reputation of the care center and will attract more patients in the future. Patient satisfaction can also be used as a measure of competitiveness for stimulating the medical team. Recent research has shown that satisfaction with quality health services and care can effectively increase life expectancy and improve the quality of patient life (Dagger \& Sweeney, 2006). According to a research conducted among Asian countries on patient satisfaction with health services and overall satisfaction with the health care facilities such as hospitals, the overall satisfaction level was significantly high among all Asian countries under study, with the exception of China (Dayasiri \& Lekamge, 2010). Figure 3 illustrates the level of satisfaction:

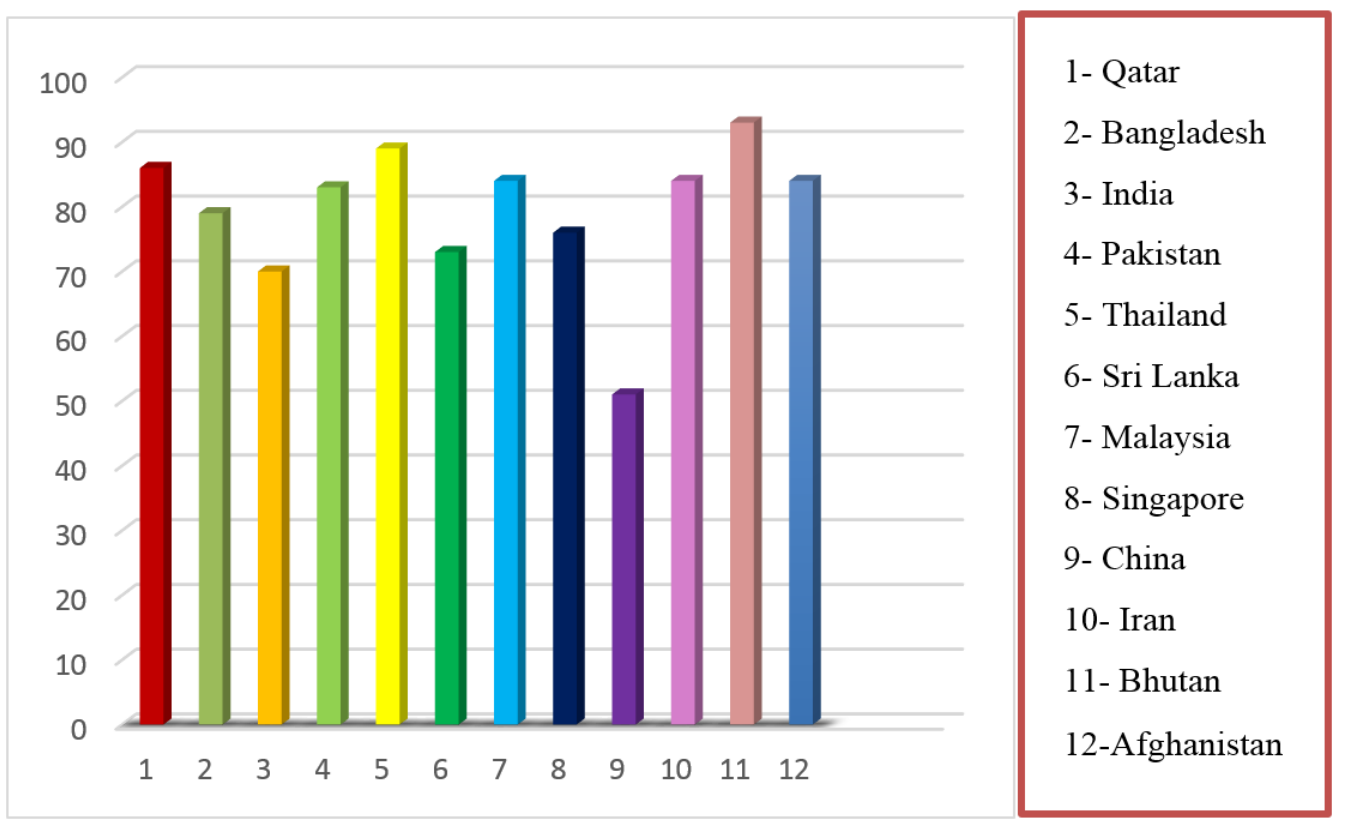

Figure 3. Level of patient satisfaction with Asian care centers (Dayasiri \& Lekamge, 2010)

\subsection{Factors Influencing Patient Satisfaction}

\subsubsection{Age}

According to a research conducted in Asia, age had no significant effect the level of patient satisfaction. But studies in Sri Lanka have shown that the level of satisfaction significantly decreased between the ages of 21 and 50. In case of China, satisfaction decreases with increasing age (Farazi et al., 2014).

\subsubsection{Gender}

According to studies gender is an influential factor in Israel and Saudi Arabia. In Israel, a more satisfaction with health care was observed in men compared to women (Biderman et al., 1994). In contrast, women in Saudi Arabia have shown more satisfaction than men. Such an effective relationship between gender and satisfaction with services and care centers was not observed in other countries under study (Al-Doghaither, 2004).

\subsubsection{Literacy}

Investigations in the United Arab Emirates (Margolis et al., 2003) and Saudi Arabia (Al-Doghaither, 2004) showed that patients who have a university degree are less satisfied than other patients. All other studies did not show such a relationship between education and satisfaction with health care centers.

\subsubsection{Socio-economic Status}

Patients from lower social classes were more satisfied with the services and care centers (Dayasiri \& Lekamge, 2010; Hansen et al., 2008). Other studies did not show such a relationship. 


\subsubsection{Type of Hospital}

There was more satisfaction in hospitals located in smaller areas. However, studies carried out in Sri Lanka (Senarath et al., 2006), China (Hansen et al., 2008) and the United Arab Emirates (Margolis et al., 2003) suggests that patients who were admitted to hospitals in urban areas showed higher satisfaction.

\subsubsection{Quality of Communication Between Medical Team and Patients}

In many studies, especially in Saudi Arabia (Al-Doghaither, 2004), Maldives (Ibrahim, 2008), Afghanistan (Hansen et al., 2008), Japan (Elleuch, 2008), Bhutan (Gurung, 2003), and Taiwan (Cheng et al., 2014), the quality of communication and interpersonal skills was a key factor in satisfaction of patients and their companions with health care and hospitals.

\subsubsection{Type of Medical Services}

In most patients the services received from doctors had a greater impact compared to services received from the rest of the medical team. In one study, patients were more satisfied with nursing services (McGivern, 1999).

\subsubsection{Technical and Infrastructural Facilities}

One of the main factors that make patients recommend a hospital to others is technical facilities and infrastructures of that care center. These factors also affected the satisfaction of their patients and clients (Cheng et al., 2014). In one of the studies the impact of these factors was even greater than the impact of interpersonal relationships and treatment (McGivern, 1999).

\subsubsection{Patient Expectations}

According to the conducted researches, patients who had lower expectations showed greater satisfaction (Mawajdeh et al., 2001).

\section{Conclusion}

Global population growth has led to higher numbers of patients and at the same time a close competition among hospitals and care centers for attracting more patients. On the other hand, medical tourism is also important from an economic point of view and Iran enjoys a favorable status for attracting foreign patients. This study suggests that various factors play a role in attracting patients and tourists to health centers. Among these factors, technical facilities and infrastructure as well as patient expectations had the greatest impact on satisfaction. Along with the expansion of media and increased public awareness, customer expectations of care centers have also increased. Thus, the main and important factors, which are even more effective than interpersonal relationships, are providing technical and infrastructural facilities consistent with the rising expectations of the clients. Also the evidence suggests that appropriate architecture and design for care environments and enhancing their quality is of particular importance for increasing the satisfaction of patients and their companions; and fitting the needs and expectations of patients and their companions result in more visits in the future and recommendations to other patients, as well as, increasing medical tourism. Of factors influencing patient satisfaction, age, gender, literacy, socio-economic status, type of hospital, the quality of communication between staff and patients, type of medical services, technical and infrastructural facilities, and patient expectations were investigated. It was also noted that one of the most important features required for hospital hoteling is the possibility of having a companion with the patient, along with an appropriate space for medical team to relax. Therefore, considering the primary and secondary design elements, the designers should allocate an appropriate space for patient companion and relaxing space for medical team. An appropriate design, considering primary elements such as light, privacy, and circulation, as well as, correct use of the relevant secondary elements such as nature, timeless, tradition, security, way-finding, contemporary, elegance, and artificial, increases patient and medical team satisfaction, which in turn improves the manner of handling and interpersonal relationships between the medical team and patients. However, the rate and manner of benefiting from these elements depend on several factors, including the views of architects and designers, urban context, and the culture of the region. Thus, appropriate design along with other factors improves the quality of patient care and the medical team performance, leading to increased life expectancy and expansion of medical tourism. For future research, a field survey can be carried out on the reciprocal impact of the mentioned primary and secondary design elements on patient and medical team satisfaction, enhanced healing, and life expectancy among patients in different hospitals in different parts of Iran.

\section{References}

(Anonymous, 2006) Medical Tourism: A Global Analysis, A Report By Tourism Research And Marketing., Retrieved March, 2015, from http://www.tram-research.com/MedicalTourism.PDF 


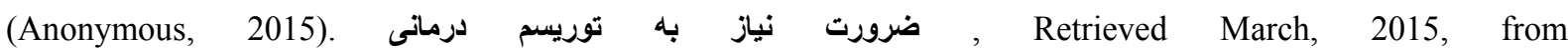
http://www.qums.ac.ir/portal/home/?102302/

Al-Doghaither, A. H. (2004). Inpatient satisfaction with physician services at King Khalid University Hospital, Riyadh, Saudi Arabia. Eastern Mediterranean Health Journal, 10(3), 358-364.

Biderman, A., Carmel, S., \& Yeheskel, A. (1994). Measuring Patient Satisfaction in Primary Care: A Joint Project of Community Representatives, Clinic Staff Members and a Social Scientist. Family Practice, 11(3), 287-291.

Dagger, T., \& Sweeney, J. C. (2006). The effects of service evaluation on behavioural intentions and quality of life. Journal of Service Research, 9, 3-19.

Dayasiri, M. B. K. C., \& Lekamge, E. L. S. (2010). Predictors of patient satisfaction with quality of health care in Asian Hospitals. Australasian Medical Journal AMJ, 3(11), 739-744.

Dietrich, K. (2004). Architectural Design Elements, Retrieved March 2015, from http:/www.kdietrich.com/thesis/d9a-research/section\%207\%20design\%20elements/section\%207\%20desig n\%20elements.pdf

Drahota, A. (2012). Hospital environments could influence patient recovery, Retrieved March, 2015, from http://www.news-medical.net/news/20120315/Hospital-environments-could-influence-patient-recovery.aspx

EI Taguri, A. (2007). Medical Tourism and the Libyan National Health Services. Department of family and community medicine, Al Fateh University, Tripoli, Libya.

Elleuch, A. (2008). Patient satisfaction in Japan. Int J Health Care Qual Assur, 21(7), 692-705.

Elsayed Metwally Amr, M. (2015). Hospital or hotel? A new way of thinking in healthcare facility Design, Hamad Medical Corporation, Doha, Qatar. Retrieved March, 2015, from http:/hospitalbuildandinfrastructure.com/?portfolio=hospital-or-hotel-a-new-way-of-thinking-in-healthcarefacility-design

Farazi, A., Eshrati, B., Ahmari Neghad, M., \& Shamsi, M. (2014). Assessment Hospital Services Quality and Satisfaction from Patients' Point of View: A Cross-sectional Study. Middle-East Journal of Scientific Research, 21(4), 652-657.

Farmahini Farahani, M., Shamsikhani, S., \& Sajadi Hezaveh, M. (2014). Patient Satisfaction With Nursing and Medical Care in Hospitals Affiliated to Arak University of Medical Sciences in 2009. Retrieved March, 2015, from http://nmsjournal.com/35110.pdf

Gurung, T. (2003). Factors influencing patient satisfaction in a free health care system in the National Referral Hospital, Thimpu, Bhutan, Health Systems Development, Chulalongkorn University, ISBN-974-9599-38-1.

Ham, C., Dixon, A., \& Brooke, B. (2012). Transforming the delivery of health and social care, The King's Fund publication, London.

Hansen, P. M., H. peters, D., Viswanathan, K., Dipankar rao, K., Mashkoor, A., \& Burnham, G. (2008). Client perceptions of the quality of primary care services in Afghanistan. International Journal for Quality in Health Care, 20, 384-391.

Ibrahim, A. (2008). Patient satisfaction with the health services at the out patients' department of Indira Ghandhi Memorial Hospital, Male, Maldives, Mahidol university.

Lee, S. (2011). Evaluating serviceability of healthcare servicescapes: Service design perspective. International Journal of Design, 5(2), 61-71.

Lunt, N., Smith, R., Exworthy, M., T. Green, S., Horsfall, D., \& Mannion, R. (2012). Medical Tourism: Treatments, Markets and Health System Implications: A scoping review. University of York, London School of Hygiene \& Tropical Medicine, Royal Holloway University of London, Sheffield Teaching Hospitals Foundation NHS Trust \& University of Birmingham.

Maguire, M., Peace, S., Nicolle, C., Marshall, R., Sims, R., Percival, J., \& Lawton, C. (2014). Kitchen living in later life: Exploring ergonomic problems, coping strategies and design solutions. International journal of design, 8(1), 73-91.

Margolis, S. A., Al-Marzouqi, S., Revel, T., \& Reed, R. L. (2003). Patient satisfaction with primary health care services in the United Arab Emirates. Int J Qual Health Care, 15(3), 241-249.

Mawajdeh, S. M., Daabseh, K. A., Nasir, M. J., \& Al-qutob, R. J. (2001). Patients' expectations and satisfaction 
in different hospitals in Irbid, Jordan. Saudi Med J., 22(7), 625-9.

McGivern, S. A. (1999). Patient satisfaction with quality of care in a hospital system in Qatar. J healthy Qual, $21(1), 28-9,32-6,41$.

Nasiripour, A. (2011). The role of the capabilities of hospitals in Tehran in the development of medical tourism. Retrieved $\quad$ March, 2015, from http://jhosp.tums.ac.ir/files/site1/user_files_3a8173/tums-A-10-25-80-671cd7f.pdf

Nedelea, A., \& George, B. P. (2008). Medical Tourism: The Next Big Thing to Come! Retrieved March, 2015, from http://ssrn.com/abstract=1264925

Newhouse, J. (1994). Frontier Estimation: How Useful a Tool for Health Economics? Journal of Health Economics, 13(3), 317-322.

Newsome, P. R. H., \& Wright, G. H. (1999). Patient management: A review of patient satisfaction: Concepts of satisfaction. British dental journal, 186, 161-165.

Pellitteri, G., \& Belvedere, F. (2010). Charateristics of the hospital buildings: Changes, Processes and Quality, Retrieved March, 2015, from http://www.aia.org/aiaucmp/groups/aia/documents/pdf/aiab087217.pdf

Perneger, T. V. (2004). Adjustment for patient characteristics in satisfaction surveys, International Journal for Quality in Health Care and Oxford University Press 2004; Volume 16, Number 6: pp. 433-435, Retrieved March, 2015, from http://intqhc.oxfordjournals.org/content/intqhe/16/6/433.full.pdf

Peterson Robert, A., \& Wilson William, R. (1992). Measuring customer satisfaction: Fact and artifact. J Acad Market Sci., 20, 61-71.

Senarath, U., Fernando, D. N., \& Rodrigo, I. (2006). Factors determining client satisfaction with hospital-based perinatal care in Sri Lanka. Trop Med Int. Health, 11(9), 1442-51.

Taherizadeh, A., (2014), طرح تحول نظام سلامت / سربرست تيم ارزياب، ارتقاء خدمات هنلينح بيمارستانها را از بهترين برنامه هاب

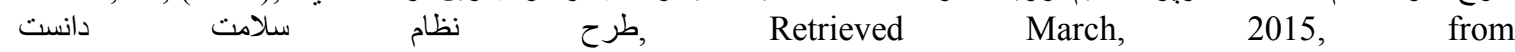
http://publicrelations.tums.ac.ir/news/detail.asp?newsID $=48407$

Tay, A. (2003). Assessing Competition in Hospital-Care Markets: The Importance of Accounting for Quality Dierentiation. RAND Journal of Economics, 34(4), 786-814.

\section{Copyrights}

Copyright for this article is retained by the author(s), with first publication rights granted to the journal.

This is an open-access article distributed under the terms and conditions of the Creative Commons Attribution license (http://creativecommons.org/licenses/by/3.0/). 